\title{
Significance of Adenosine Deaminase in Diagnosing Tuberculous Pleural Effusion in Nepalgunj Medical College Teaching Hospital Kohalpur
}

\author{
Shyam BK ${ }^{1}$, Shah SK$^{2}$, Sumit $P^{3}$, Sushil $B^{4}$, Sandeep $G^{5}$
}

\begin{abstract}
Background: Tuberculosis (TB) is a major public health problem in developing countries including Nepal. One of the common presentations of TB is pleural effusion. The diagnosis of tubercular pleural effusion can be difficult because of the low rate of detecting tubercular bacilli by direct stain and culture of pleural fluid for acid-fast bacilli (AFB). Pleural biopsy can be useful but is invasive and requires experts. In this context, pleural fluid Adenosine Deaminase (ADA) level has been proposed as easy, cheap and highly sensitive test for diagnosis of TB pleural effusion. Objectives: The present study was undertaken to define the role of pleural fluid ADA value in accurate diagnosis of Tubercular pleural effusion. Methods: A Prospective analysis of 68 patients admitted in Nepalgunj Medical College teaching Hospital was done from January 2014 to December 2015 with pleural effusion. Pleural fluid ADA level was evaluated in all patients, and significance of pleural fluid ADA level in TB pleural effusion was studied. Results: Age of patients were between 20 to 80 years, with the minimum being 20 years and maximum being 79 years. In this study $85 \%$ of cases had pleural effusion due to tuberculosis. Out of the 68 patients with pleural effusion, 58(85\%) were finally diagnosed to be due to tuberculosis, 2 were diagnosed to be due to malignancy, 4 due to pneumonia leading to parapneumonic effusion, 1 due to congestive heart failure and 3 due to nephrotic syndrome. Conclusion: It is difficult to diagnose TB pleural effusion by other conventional methods, as it has also been shown in our study also. Previous literatures have also mentioned AFB detection rate to be low from pleural fluid sample. Determination of ADA is a cheap and easy test which we now consider in the early routine evaluation of patients with pleural effusions, particularly if diagnosis of tuberculosis is suspected and in places where prevalence of the disease is still high as is in our country. The other method considered for diagnosing TB pleural effusion is pleural biopsy which is invasive blind procedure and requires high expertise as well.
\end{abstract}

Key words: Adenosine deaminase, tuberculosis, pleural effusion, $A F B$

\section{INTRODUCTION}

Pleural effusion is a common chest problem, yet it is difficult to establish the aetiological diagnosis in as many as $20 \%$ cases in spite of good history, thorough clinical, radiological, full examination of aspirated fluid and pleural biopsy ${ }^{1}$. The initial event in the pathogenesis of primary TB pleural effusion is the rupture of subpleural caseous focus in the lung'. Tuberculous pleural effusion is thought to result from a delayed hypersensitivity reaction in response to the presence of mycobacterial antigens in the pleural space that follows this rupture ${ }^{3}$. The accumulation of fluid in pleural cavity results due to increased capillary permeability as well as due to impairment of lymphatic clearance of exudative fluid from pleural cavity due to occlusion of pleural stomata. The

1. Dr. Shyam B. K.

2. Dr. Sujeet Kumar Shah

3. Dr. Sumit Pandey

4. Dr. Sushil Baral

5. Dr. Sandeep Gupta

Address for correspondence:

Dr. Shyam B. K.

Department of Medicine

Nepalgunj Medical College Teaching Hospital,

Kohalpur, Banke, Nepal

Email: dr.shyamyp@gmail.com diagnosis of tuberculous pleural effusion can be difficult because of the low sensitivity of various diagnostic methods. Lymphocytic exudate seen in tuberculous pleural effusion also can occur in other diseases such as malignancy and collagen vascular diseases. Mycobacterium tuberculosis in pleural fluid is scanty and rarely observed on direct examination by AFB staining. Cultures for AFB in TB pleural effusion are positive in only 20 to $30 \%$ of pleural fluid samples and in 50 to $80 \%$ of pleural biopsy specimens ${ }^{4}$.

The sensitivity of polymerase chain reaction for active disease is $78 \%{ }^{5}$. The cutaneous response to purified protein derivative (Mantoux test) may also be negative in one third of the patients ${ }^{6}$. In this context, attempts have been made to identify markers which allow more rapid and accurate diagnosis. One such marker is adenosine deaminase (ADA), which has been proposed to be a useful diagnostic marker for tuberculous disease in pleura, pericardium, and peritoneum ${ }^{7}$. Several reports have suggested that an elevated pleural fluid ADA level predicts tuberculous pleurisy with a sensitivity of 90 to $100 \%$ and a specificity of 89 to $100 \%$. ADA is an enzyme in the purine salvage pathway that catalyses conversion of adenosine and deoxyadenosine to inosine and deoxyinosine with the release of ammonia ${ }^{9}$. Its distribution in the human organ is ubiquitous, but its physiologic role is especially important in lymphoid tissue. $^{10}$ 


\section{Material and Methods}

A Prospective analysis of 68 patients of pleural effusion from January 2014 to December 2015 was done. Both male and female, above the age of 15 years who were admitted in the Nepalgunj Medical College Teaching Hospital Kohalpur Banke Nepal. Patients in whom history of typhoid fever, acute viral hepatitis and active cirrhosis were present, were excluded. Detailed history was taken and thorough clinical examination was done in each and every patients and they were then subjected to a batteries of investigation which included routine haemogram, urine examination, skiagram chest PA view, sputum smear examination for AFB and sputum culture for mycobacterium tuberculosis, pleural fluid for protein, glucose, cell count, malignant cells, Gram's stain, pleural fluid examination for AFB, pleural fluid culture for Mycobacterium tuberculosis the other relevant investigation as per need of cases. ADA was measured in pleural fluid by colorimetric method of Guisti and Galanti ${ }^{11}$. Patients with relevant clinical history and examination findings with exudative pleural fluid supported by Mantoux test as well as all those patients with ADA value $>40 \mathrm{U} / \mathrm{L}$ were provisionally diagnosed to have TB pleural effusion and standard ATT regimen was started.

\section{RESULTS}

Age of patients was between 20 to 80 years years, with the minimum being 20 years and maximum being 79 years. Among 68 patient 32(47.05\%) were male and 36(52.94\%) were female shown in Table-I. Age of the patient is shown in Figure 1. The signs and symptoms elicited in patients are as shown in the Table-II. Of 68 patients with pleural effusion, 58 were finally diagnosed to be due to tuberculosis, 2 were diagnosed to be due to malignancy, 4 due to pneumonia leading to parapneumonic effusion, 1 due to congestive heart failure and 3 due to nephrotic syndrome. Number of sputum positive for Acid Fast Bacilli is shown in Figure 2. Analyzing pleural fluid by Light's criteria, all cases with TB, malignancy and pneumonia had exudative effusion, all cases with CHF had transudative effusion whereas $50 \%$ of cases of nephrotic syndrome had exudative and $50 \%$ had transudative effusion.

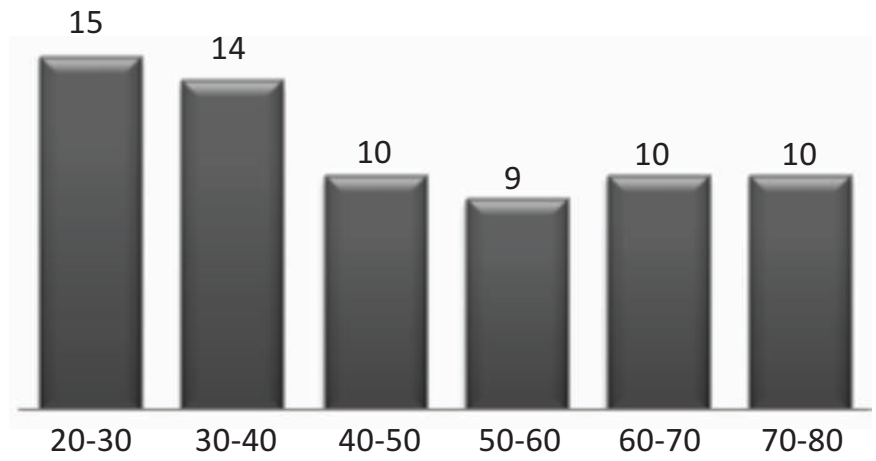

Figure 1: Age distribution

\begin{tabular}{|c|c|c|}
\hline Sex & No. of patient & Percentage \\
\hline Male & 32 & $47.05 \%$ \\
\hline Female & 36 & $52.94 \%$ \\
\hline
\end{tabular}

Table I: Sex distribution

\begin{tabular}{|c|c|c|}
\hline Clinical features & No. of cases & Percentage \\
\hline H/O Smoking & 52 & $76.47 \%$ \\
\hline Fever & 25 & $36.76 \%$ \\
\hline Cough & 61 & $89.70 \%$ \\
\hline SOB & 63 & $92.64 \%$ \\
\hline Chest pain & 38 & $55.88 \%$ \\
\hline Hemoptysis & 15 & $22.05 \%$ \\
\hline Others & 30 & $44.11 \%$ \\
\hline
\end{tabular}

Table II: Signs and symptoms

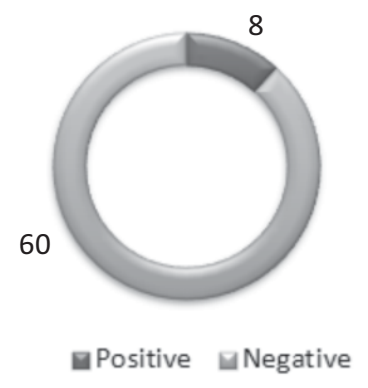

Figure 2: Showing sputum positive and negative cases

Out of the 2 cases which were diagnosed as malignancy 1 patient had positive pleural fluid cytology for malignant cells. All cases provisionally diagnosed as TB (58 cases) were put on anti-tubercular treatment as per DOTS, of which 10 cases were lost follow up, 2 did not respond to treatment and the remaining 46 cases were cured. There were 58 cases with pleural fluid ADA value more than $40 \mathrm{U} / \mathrm{L}$, out of which majority (23 cases) had ADA more than $60 \mathrm{U} / \mathrm{L}$. The values of ADA in pleural fluid in all patient is shown in figure 3 .

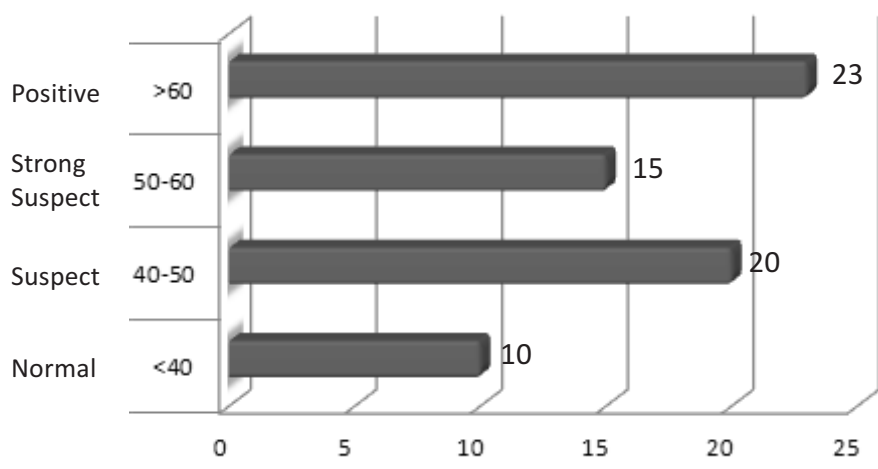

Figure 3: Value of ADA in all cases 


\section{DISCUSSION}

In this study $85 \%$ of cases had pleural effusion due to tuberculosis. All cases of TB pleural effusion were exudative which is explained by the pathogenesis of formation of effusion in TB. Considering pleural fluid ADA level in different conditions, ADA level was high (more than 40U/L) in 58 cases which was $85 \%$ as compared in parapneumonic effusion, in malignancy and in cases with miscellaneous diagnosis. Similar findings have been found in several previous studies. Ocana et $\mathrm{al}^{12}$ found mean pleural fluid ADA level to be $92.43 \pm 29.43 \mathrm{U} / \mathrm{L}$ in TB pleural effusion. Valdes et al $^{13}$ found pleural fluid ADA level in TB pleural effusion to be $111.1 \pm 36.6 \mathrm{U} / \mathrm{L}$. Thus, high ADA value in pleural fluid can diagnose TB with high accuracy. Several studies have defined several cut-off values for diagnosis of TB pleural effusion ranging from 40 to $70 \mathrm{U} / \mathrm{L}$. Our study also shows that there are no other causes of pleural effusion for ADA level $>40 \mathrm{U} / \mathrm{L}$ than TB, except for few cases due to parapneumonic effusion. Ocana et al found $97 \%$ sensitivity and $100 \%$ specificity for value more than $45 \mathrm{U} / \mathrm{L}$. Banales et al found ADA level of $>70 \mathrm{U} / \mathrm{L}$ to be $98 \%$ sensitive and $96 \%$ specific. ${ }^{14} \mathrm{DE}$ Oliveira et al found ADA level of $>40 \mathrm{U} / \mathrm{I}$ to be $90.7 \%$ sensitive and $97.7 \%$ specific $^{15}$.

Abnormally high levels of pleural fluid ADA level in all patients with TB pleural effusion indicated pleural fluid ADA could be a good diagnostic marker for TB pleural effusion. In our study, patients diagnosed as TB, depending on relevant clinical history and high ADA level in pleural fluid, were put on standard ATT regimen and almost all were cured. This observation provides evidence in support of using pleural fluid ADA level as a diagnostic marker for TB pleural effusion.

In conclusion, determination of ADA is a cheap and easy test, which we now consider in the early routine evaluation of patients with pleural effusions, particularly if diagnosis of tuberculosis is suspected and in places where prevalence of the disease is still high as is in our country. By using ADA as a marker for the diagnosis of tuberculous pleural effusion, the accurate diagnosis can be made in more than 95 percent of cases and it helps avoid invasive test like pleural biopsy as well.

\section{CONCLUSION}

ADA is an enzyme in the purine salvage pathway that catalyses conversion of adenosine and deoxyadenosine to inosine and deoxyinosine with the release of ammonia. Its distribution in the human organ is ubiquitous, but its physiologic role is especially important in lymphoid tissue. ADA is a predominant $T$ lymphocyte enzyme, with variation according to cellular differentiation, and its plasma activity is high in diseases where cellular immunity is stimulated. It is well known that tuberculosis is one of these diseases. The ADA activity is raised in lymphocytic pleural effusions of tuberculous origin only. ADA analysis is a sensitive marker of tuberculous pleural effusion, even in HIV patients with very low CD4 counts.

\section{REFERENCES}

1. Kataria Y P, Imtiaz K. Adenosine deaminase in the diagnosis of tuberculous pleural effusion. Chest 2001, 2: 298-300.

2. Stead WW, Eichenholz A, Strauss HK. Operative and pathologic findings in twenty-four patients with syndrome of idiopathic pleurisy with effusion, presumably tuberculosis. Amer Rev Tuberc 1955; 71: 473-502.

3. Leibowitz S, Kennedy L, Lessof $\mathrm{MH}$. The tuberculin reaction in the pleural cavity and its suppression by anti lymphocyte serum. Brit J Exp Pathol 1973; 54: 152-62.

4. Jay SJ. Diagnostic procedures for pleural diseases: symposium on pleural diseases. Clin Chest Med 1985; 6: 33-48.

5. De Wit D, Maartens G, Steyn L. A comparative study of the polymerase chain reaction and conventional procedures for the diagnosis of tuberculous pleural effusion. Tubercle 1992; 73: 2627.

6. Valdes L, Alvarez D, San Jose E et al. Tuberculous pleurisy, a study of 254 patients. Arch Intern Med 1998; 158: 2017-21.

7. Segura RM, Pascual C, Ocafia I et al. Adenosine deaminase in body fluids: a useful diagnostic tool in tuberculosis. ClinBiochem 1989; 22: 141-8.

8. Roth BJ. Searching for tuberculosis in the pleural space. Chest 1999; 116: 3-5.

9. Martin DW Jr, Gelfand EW. Biochemistry of diseases of iinmunodevelopment. Ann Rev Biochem 1981; 50: 845-77.

10. Van Der Weyden MB, Kelley WN. Human adenosine deaminase: distribution and properties. J Biol Chem 1976; 251: 5448-56.

11. Guisti G, Galanti B. Colorimetric method In: Bergmeyer HU,ed. Method of Enzymatic analysis 3rd ed. Berlin, Germany.

12. Ocaña I, Martinez-Vazquez JM, Segura RM, Fernandez-De-Sevilla T, Capdevila JA. Adenosine deaminase in pleural fluids: test for diagnosis of tuberculous pleural effusion. Chest 1983; 84: 51-3.

13. Valdes $L$, Alvarez $D$, Jose ES et al. Value of adenosine deaminase in the diagnosis of tuberculous pleural effusions in young patients in a region of high prevalence of tuberculosis. Thorax 1995; 50: 600-3.

14. Bañales JL, Salazar-Lezama, Pineda PR, Fitzgerald JM,Rubio H, Selman M. Adenosine deaminase in the diagnosis of tuberculous pleural effusions. A report of 218 patients and review of the literature. Chest 1991; 99: 355-7.

15. DE Oliveira HG, Rossatto E, Prolla JC. Pleural fl uid adenosine deaminase and lymphocyte proportion: clinical usefulness in the diagnosis of tuberculosis. Cytopathol 1994; 5: 27-32. 